\title{
Descentralizando TI com a adoção do End-User Development: dois estudos de caso
}

\author{
Claudia de O. Melo ${ }^{1}$, Rejane M. da C. Figueiredo ${ }^{2}$, Elaine Venson ${ }^{2}$ \\ ${ }^{1}$ Departamento de Ciência da Computação (CIC-UnB) \\ Brasília - DF - Brasil \\ ${ }^{2}$ Faculdade do Gama (FGA-UnB) \\ Brasília - DF - Brasil \\ \{claudia.melo, rejanecosta, elainevenson\}@unb.br
}

\begin{abstract}
Context: Digital transformation is changing the industry and the way we use Information Technology (IT). Software is mostly written by end-users with business knowledge willing to achieve theirs goals through IT, although established IT governance is not always appropriate. Understanding how to organize the decentralization of the IT function to prepare the organization for the challenges and opportunities of the 21st century is key. Goal: This paper aims to investigate, from a socio-technical perspective, how organizations decentralize IT through End-User Development (EUD). Method: Two case studies have been conducted in organizations trying to decentralize IT through EUD for, at least, 3 years. A conceptual framework has been described to allow case analysis. Results: IT governancematurity was an important factor that enabled decentralization through the EUD, although both cases did not reach a high degree of decentralization. Shadow IT remained in both cases. Both organizations were not focused on innovation during the decentralization initiatives, although this is a fundamental vision of for the IT function in the 21 st century.
\end{abstract}

Resumo. Contexto: A transformação digital está mudando a indústria e a maneira como usamos a tecnologia da informação (TI). A maior parte dos programas já são escritos por usuários finais com conhecimento do negócio que precisam cumprir objetivos com o uso da TI, embora a governança estabelecida nem sempre seja adequada. A compreensão de como organizar a descentralização da função de TI para usuários finais, de modo a preparar a organização para os desafios e oportunidades do século 21, torna-se chave. Objetivo: Este trabalho tem como objetivo investigar, sob uma ótica socio-técnica, como organizações descentralizam a TI por meio de End-User Development (EUD). Método: Foram conduzidos dois estudos de caso em organizações que buscam a descentralização por EUD há pelo menos 3 anos. Foi descrito um framework conceitual para análise dos casos. Resultados: A maturidade prévia em governança da TI foi fator importante na capacidade de descentralização através de EUD, embora ambos casos não tenham atingido alto grau de descentralização. A TI sombra permaneceu em ambos os casos. Ambas organizações não demonstraram buscar inovação com as iniciativas de descentralização, embora esta seja uma visão fundamental da função de TI no século 21 . 


\section{Introdução}

A transformação digital está mudando a indústria e a maneira como usamos a tecnologia da informação (TI) [Bygstad 2016]. Duas tendências atuais geram as forças dessa transformação. Por um lado, os sistemas de TI estão cada vez maiores e mais interconectados. Por outro, o uso crescente de equipamentos pessoais móveis na vida profissional vem desafiando a hegemonia dos departamentos de TI. Os movimentos "traga seu próprio dispositivo" e "faça você mesmo" estão se tornando rapidamente um fato aceitável na vida corporativa, cuja tendência é denominada consumerização e gera um conjunto de novos desafios sobre uso, segurança e governança de TI [Niehaves et al. 2012, Köffer et al. 2014].

De fato, a maior parte dos programas não são escritos por profissionais de desenvolvimento de software, mas por pessoas com conhecimento do negócio que precisam cumprir objetivos com o uso da TI [Ko et al. 2011], com ou sem o conhecimento e suporte do departamento de TI. Quando desenvolvidos sem a conscientização, aceitação, conhecimento ou suporte de um departamento de TI, são denominados "TI invisível"ou "TI Sombra"(Shadow IT) [Silic and Back 2014]. Já o Desenvolvimento por Usuário Final (End-User Development, ou EUD) ocorre quando há o desenvolvimento, modificação ou aperfeiçoamento de aplicações de sistemas de informação por usuários finais para uso individual, departamental ou organizacional [Barker and Fiedler 2011], com conhecimento e suporte do departamento de TI [Zimmermann et al. 2014].

Se antes esses usuários já desenvolviam grande parte das soluções, agora são vistos como centrais no novo regime de conhecimento baseado em inovação digital [Bygstad 2016]. De fato, a inovação esperada na Economia Digital depende dessa rápida apropriação tecnológica por parte de usuários comuns, dado que a inovação pode surgir a partir de qualquer pessoa. Soluções criadas por usuários finais, antes vistas como um problema a ser gerenciado pelas organizações, tornam-se agora um recurso estratégico [Dittrich et al. 2017]. A tecnologia e todos seus stakeholders passaram a ter uma função e conjunto de competências muito mais importantes, pois geram novo valor de negócio [Andriole 2015, Köffer et al. 2015].

A transformação digital vem mudando a forma como a governança é definida e gerenciada em muitas organizações [Andriole 2015]. Até o século 20, a gestão tecnológica se concentrou majoritariamente em definição de padrões e gerenciamento centralizado. Na economia do século 21, dirigida pela inovação digital, a ênfase nesse paradigma perdeu o sentido. A TI não é mais propriedade de um departamento central, mas uma função inerente a toda a organização. Assim, algumas organizações estão experimentando a descentralização da gestão de TI, passando a modelos federados e até participatórios [Andriole 2015].

A compreensão de como descentralizar a função de TI de modo a preparar a organização para os desafios e oportunidades do século 21 torna-se chave. Pesquisas recentes investigam diferentes aspectos sobre modelos de governança do EUD, assim como do fenômeno da TI Sombra, sob o ponto de vista de alocação das responsabilidades [Winkler and Brown 2013, Zimmermann et al. 2016], alinhamento entre usuários, TI e organização [Chua et al. 2014], colaboração [Ferneley 2007] e relações de poder e risco [Fürstenau et al. 2017]. Um aspecto fundamental de investigação para apoiar organizações que buscam descentralizar TI através de EUD é compreender as interações 
entre os diferentes stakeholders e tecnologias envolvidos para o sucesso de uma iniciativa em nível organizacional.

Este trabalho tem como objetivo investigar, sob uma ótica socio-técnica, a questão de pesquisa (QP) “Como organizações descentralizam a TI por meio de EUD?". Para responder à questão, foram conduzidos dois estudos de caso em organizações que buscam ampliar sua capacidade de lidar com TI com a adoção da descentralização por EUD há pelo menos 3 anos. Para analisar os casos, derivamos um framework conceitual baseado em um paradigma socio-técnico de meta-design de EUD proposta em [Fischer et al. 2004] e também nos mecanismos gerais de governança de TI propostos em [Weill and Ross 2004].

As principais contribuições deste artigo são:

- A descrição dos conceitos relacionados ao EUD, contextualizando-os em uma nova era de Governança de TI, na Seção 2;

- A apresentação de um framework conceitual para analisar a descentralização de TI pela adoção de EUD, derivado de dois trabalhos sólidos da área de governança e de EUD, na Seção 3;

- O uso do framework conceitual para análise de dois estudos de caso para investigar como organizações descentralizam a TI por meio de EUD, na Seção 4;

- A descrição da abordagem de descentralização utilizada por cada uma das organizações investigadas e uma síntese comparada ao framework conceitual, na Seção 5;

- Uma discussão sobre os elementos relevantes encontrados nos dois casos frente ao framework conceitual e a literatura, na Seção 6.

\section{Desenvolvimento teórico}

\subsection{Desenvolvimento por Usuário Final (EUD)}

O desenvolvimento por usuário final (End-User Development - EUD) é realizado por usuários finais e não-especialistas em programação de aplicações que desenvolvem ou adaptam sistemas de acordo com suas necessidades, sejam profissionais, educacionais ou para lazer [Lieberman et al. 2006]. EUD é, mais especificamente, o desenvolvimento, modificação ou aperfeiçoamento de aplicações de sistemas de informação por usuários finais para uso individual, departamental ou organizacional [Barker and Fiedler 2011].

Usuários finais desenvolvedores podem criar software em uma variedade de domínios, de aplicações domésticas a aplicações financeiras, científicas, administrativas ou de recursos humanos [Figueiredo et al. 2017]. As atividades e ferramentas utilizadas para o desenvolvimento das soluções de software por usuários são diferentes, conforme os objetivos dos tipos de usuários.

Outras abordagens similares a EUD são Rapid Application Development (RAD) e Low Code. Esse último termo vem sendo cunhado recentemente pela indústria para caracterizar plataformas que permitam a entrega rápida de aplicativos de negócios com um mínimo de codificação manual e investimento inicial de configuração, treinamento e implantação [Richardson and Rymer 2016].

O objetivo de se adotar a abordagem EUD como estratégia organizacional, que inclui processos e ferramentas, é reduzir o fardo de aprendizagem e, ao mesmo tempo, 
dar instrumentos poderosos aos funcionários para lidar com inúmeros tipos de problemas envolvendo demanda de TI [Fischer et al. 2004]. Dado que algum fardo de aprendizagem sempre estará presente, as ferramentas precisam motivar seus usuários. O sucesso do EUD depende de um equilíbrio entre a motivação do usuário, ferramentas efetivas e suporte de gerenciamento.

As pesquisas da comunidade de EUD também descrevem uma transformação cultural fundamental no papel do usuário, que deixa de ser um consumidor passivo de produtos produzidos por outros, para um usuário empoderado para participar ativamente de atividades de caráter significativo [von Hippel 2005, Fischer 2009, Maceli 2017]. EUD é componente essencial dessa transformação. Seu impacto, de fato, pode ser muito mais amplo: essa transformação representa uma mudança e uma nova oportunidade para a produção social, para a colaboração em massa, para a vida cívica e política e para a educação [Fischer 2009].

A adoção de EUD também envolve alguns riscos. Em revisão de literatura conduzida por [Figueiredo et al. 2017], foram identificados os principais riscos da adoção de EUD. Dentre eles estão a baixa qualidade das aplicações desenvolvidas, perda do controle do desenvolvimento da aplicação, utilização ineficaz dos recursos, ameaça à segurança dos dados, ameaça à integridade dos dados, resolver o problema errado e abandono da aplicação pelos usuários utilizadores.

$\mathrm{Na}$ revisão de estado da arte de EUD, [Ko et al. 2011] apontam que alguns trabalhos recentes sobre o EUD estão se movendo em várias direções ao mesmo tempo: para mais plataformas e paradigmas, incluindo a web e dispositivos móveis; para explorar boas práticas da engenharia de software, incluindo especificação e reutilização de código; para um foco mais amplo em domínios de aplicação, ao invés de paradigma de linguagem único. Além disso, os autores afirmam que a Web tem se tornado uma plataforma dominante para a aprendizagem da programação pelos usuários finais, bem como, para apoiar suas atividades de desenvolvimento do EUD.

\subsection{Governança de TI na Era Digital}

A governança de TI pode ser definida como "a distribuição de direitos e responsabilidades de decisão de TI entre as diferentes partes interessadas na empresa e define os procedimentos e mecanismos para elaborar e monitorar decisões estratégicas de TI" [Peterson 2004]. Ela define os papéis e responsabilidades para tomar decisões relacionadas à TI, além de processos efetivos de tomada de decisão e mecanismos de avaliação de desempenho.

No entanto, na era digital, produtos e serviços tornam-se cada vez mais digitalizados, desfigurando os limites entre os processos de TI e negócios (por exemplo, marketing, vendas, fabricação) [Bharadwaj et al. 2013]. Muitas decisões, portanto, ocorrem em conjunto em equipes multifuncionais em oposição à tomada de decisão autônoma de nível funcional ou bilateral. O pensamento da função de TI submetida à autoridade de negócios muda à medida que ela torna-se a forma comum de entregar produtos e serviços [DeLone et al. 2017].

A alta velocidade do desenvolvimento tecnológico incentiva as empresas a desenvolver princípios de governança para simplificar e acelerar os processos de tomada de decisão relacionados à TI. Essas tendências digitais sugerem que os modelos de 
governança de TI baseados na separação distinta das responsabilidades funcionais e na tomada de decisão inerentemente hierárquica talvez precisem ser revisados [DeLone et al. 2017].

A governança está mudando, o controle é um conceito que se transforma em colaboração e participação, e a governança participativa substituirá as rígidas estruturas e processos de governança convencionais do século 20 e estruturas ainda mais abertas e federadas do início do século XXI. A governança participatória reconhece a expansão do número de stakeholders da governança para fora da organização, a comoditização da tecnologia, a consumerização e a crescente prática de terceirização de tecnologia operacional, estratégica e emergente. A literatura também sugere que a nova oportunidade de alinhamento da tecnologia empresarial é a partir da governança participatória [Andriole 2015].

\section{Framework conceitual para analisar descentralização de TI pela abordagem EUD}

Para que seja possível descentralizar a função de TI pela abordagem EUD, é necessário investigar tanto os mecanismos de descentralização da governança quanto os mecanismos habilitadores de EUD. EUD já pressupõe algum nível de descentralização, visto que se espera o maior empoderamento dos usuários finais. Esse empoderamento gerará conflitos com as estruturas de governança existentes, que podem ser frutíferos e gerar novas estruturas adaptadas ou não.

Para analisar as dinâmicas de descentralização de TI através de EUD, derivou-se um framework conceitual baseado em dois trabalhos sólidos da área de governança e de EUD. A base do modelo é dada pela visão sócio-técnica de EUD proposta em [Fischer et al. 2004]. O framework também inclui os mecanismos gerais de governança de TI propostos em [Weill and Ross 2004].

O framework descreve as diversas forças que atuam na organização em busca do sucesso de iniciativas EUD (Figura 1), detalhadas nas próximas seções.

\subsection{Motivadores do usuário}

Compreendem a capacitação para poder completar um trabalho de forma mais eficaz, a velocidade de desenvolvimento, a flexibilidade e o controle local para que a programação possa ser "sob demanda". Um dos benefícios é a eliminação de possíveis erros de comunicação de requisitos para engenheiros de software especializados, evitando assim a frustração com o serviço prestado pelo departamento de TI.

Histórias de sucesso podem criar capital motivacional para ajudar os usuários sobre a curva de aprendizado até que os benefícios reais cheguem na forma de aplicativos de trabalho. A motivação do usuário deve ser encorajada durante os estágios iniciais da adoção por meio de suporte da gestão, treinamento e forças-tarefa para difundir melhores práticas e conhecimentos especializados. Isso remedia custos dos usuários, como selecionar a tecnologia apropriada, instalá-la e aprendê-la, programá-la e depurá-la.

\subsection{Questões Gerenciais e Contextuais}

Uma série de questões de contexto e gerenciamento influenciam o equilíbrio entre custos e benefícios. Por exemplo, a abordagem EUD pode ser perigosa em domínios de segurança 


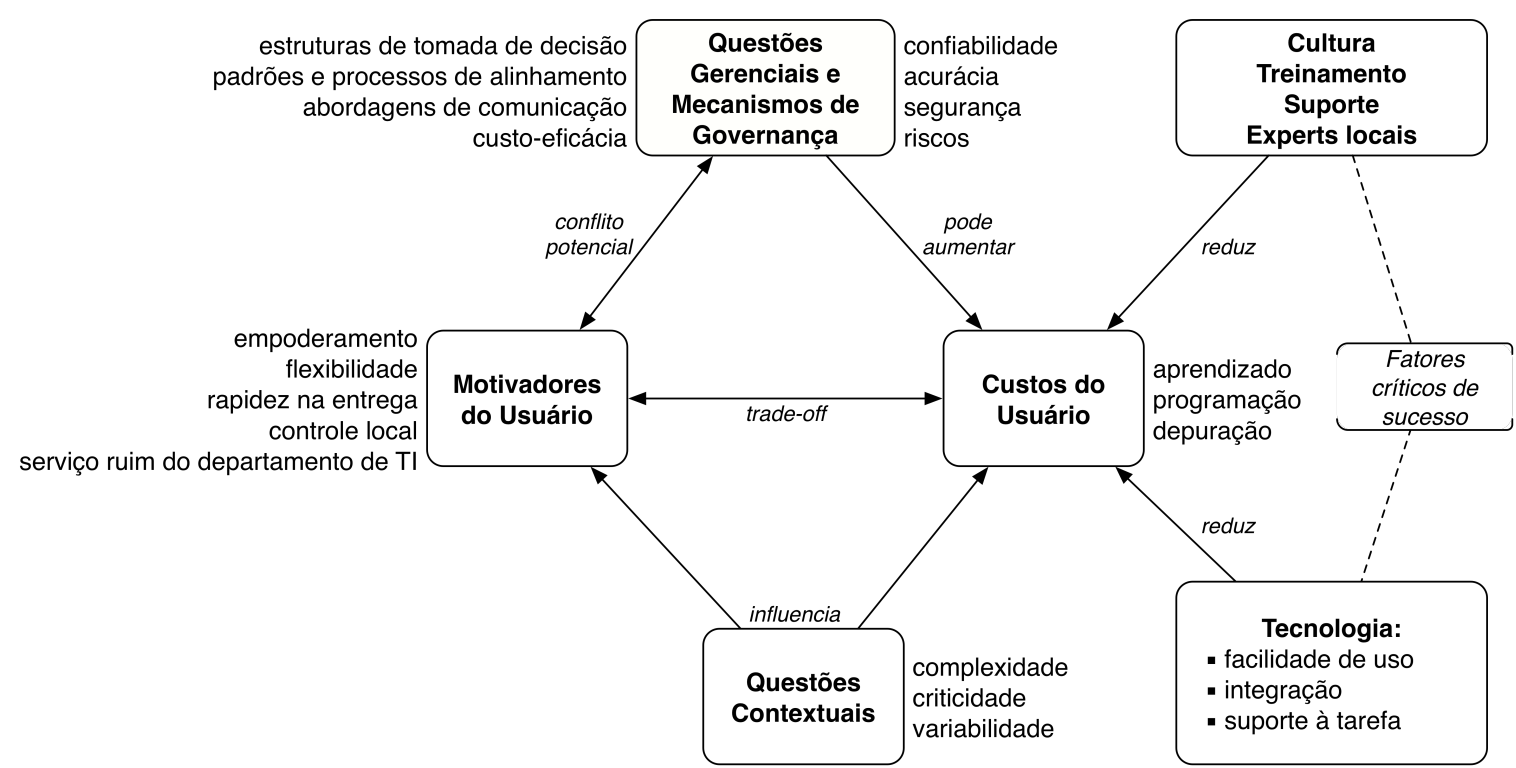

Figura 1. Framework conceitual adaptado de [Fischer et al. 2004] e [Weill and Ross 2004]

crítica, onde o software precisa ser confiável e preciso. Os custos do usuário podem ser afetados significativamente pela escala e complexidade do domínio, portanto, domínios mais seguros e menos complexos devem ser selecionados para EUD. A mudança de domínio pode ser um motivador para a adoção do EUD, uma vez que os usuários finais podem responder aos requisitos em rápida evolução, mais rapidamente do que o desenvolvimento tradicional. No entanto, mudanças rápidas podem levar a um software descartável e a um esforço de desenvolvimento perdido.

As questões de gestão incluem riscos associados à EUD, percebidos pelos departamentos de TI como a criação de software não confiável e não sustentável. Outros riscos são informações imprecisas, segurança com maior exposição à ataques. O conflito entre o gerenciamento de TI e os usuários finais sobre o poder, autoridade e controle de sistemas de TI pode ser uma força produtiva para a mudança ou pode levar à interrupção, desconfiança e falha.

Padrões e controle de usuários finais levam a um desenvolvimento mais econômico e menos desperdício de software não confiável [Fischer et al. 2004]. No entanto, o controle rígido pode gerar ressentimento entre os usuários finais. O conflito de controle e poder entre usuários e departamento de TI sempre vai existir. No entanto, interações construtivas visando apoiar e treinar usuários promove o sucesso das ações, encoraja a responsabilidade das partes e possibilita o controle por liderança.

\subsubsection{Mecanismos de governança de TI}

O modelo original de Fischer et al. [Fischer et al. 2004] considera poder, controle e responsabilidade parte das questões gerenciais, mas não os detalham. Por serem de vital importância no entendimento da descentralização da função de TI, o framework incorpora os mecanismos de governança de TI [Weill and Ross 2004, Haes and Grembergen 2009] 
que não apenas detalham poder, controle e responsabilidade, mas também são considerados necessários para uma governança bem sucedida [Frey and Buxmann 2011, Ping-Ju Wu et al. 2015].

Mecanismos de governança objetivam encorajar a congruência com a missão, a estratégia, os valores, as normas e a cultura da organização, criando condições favoráveis para os comportamentos de TI desejados. A existência desses mecanismos é muitas vezes indicativo de uma capacidade sofisticada de gerenciamento de TI e de negócios [Karimi et al. 2000, Bradley et al. 2012, Ping-Ju Wu et al. 2015].

De modo geral, pode-se entender que a governança bem-sucedida de TI implementa três tipos diferentes de mecanismos: 1) estruturas de tomada de decisão, 2) processos de alinhamento e 3) abordagens de comunicação [Weill and Ross 2004].

Estruturas de tomada de decisão referem-se ao grau em que a organização estabeleceu unidades organizacionais e funções responsáveis pela tomada de decisões de TI, como comitês e papéis que localizam as responsabilidades de tomada de decisão [Weill and Ross 2004, Haes and Grembergen 2009]. Nesse sentido, o framework considera três possíveis classificações de como organizações tomam decisões de forma mais ou menos centralizada: centralizado, federado, descentralizado e participatório.

Em organizações de TI centralizadas, os direitos de decisão envolvidos na aquisição, implantação e suporte de tecnologia pertencem a um grupo central que reporta a um executivo corporativo. Em organizações federadas, os direitos são coordenados em todo o grupo corporativo de TI, as unidades de negócios e até mesmo funções corporativas específicas [Frey and Buxmann 2011, Andriole 2015]. Já nas organizações descentralizadas, os direitos de decisão são compartilhados em toda a empresa e unidades de negócios. Por fim, em organizações participatórias, a tomada de decisões inclui stakeholders externos à organização, como fornecedores de hardware, software e serviços, parceiros e fornecedores, ou qualquer pessoa que contribua para o sucesso da organização. Esse seria o maior grau de descentralização considerado no framework.

Processos de alinhamento referem-se ao grau em que a organização estabeleceu processos formais para monitorar e garantir que as políticas de TI sejam consistentes com as necessidades do negócio. Acordos de nível de serviço e monitoramento do valor gerado são exemplos de como as organizações se forçam a avaliar continuamente o retorno real obtido através da TI [Weill and Ross 2005].

As abordagens de comunicação referem-se ao grau em que a organização estabeleceu canais para garantir comunicação e disseminação dos princípios de governança de TI. Maior comunicação geralmente significa mais governança efetiva [Weill and Ross 2005]. A administração pode comunicar os processos de governança de várias formas: anúncios gerais; a instituição de comissões formais; comunicações regulares do escritório do CIO ou do escritório de governança de TI; sessões individuais; Intranets etc.

\subsection{Fatores críticos de sucesso}

Os fatores de sucesso dependem do domínio. Em uma cultura de usuários finais motivados e baixa influência gerencial, o sucesso é simplesmente uma questão de usuários levando o desenvolvimento em suas próprias mãos, muitas vezes usando linguagens de programação 
padrão. No entanto, na maioria dos domínios empresariais, o treinamento, o suporte técnico e de gerenciamento são vitais para ajudar o EUD a prosperar.

Em uma cultura de cooperação, há compartilhamento de responsabilidade pelo desenvolvimento de soluções precisas e efetivas. Especialistas locais da comunidade de usuários finais disseminam conhecimentos e conselhos, embora os usuários avançados possam ser propensos a migrar para o lado errado do "nós" e "eles" (departamento de TI). Por fim, a tecnologia deve fornecer uma integração fácil com outros sistemas de informação e suporte otimizado para tarefas EUD.

\section{Método de Pesquisa}

Para investigar nossa questão de pesquisa "Como organizações descentralizam a TI por meio de EUD?", foram realizados dois estudos de caso [Yin 2009] em organizações públicas federais. Estudos de caso são adequados para investigações socio-técnicas [Benbasat et al. 1987, Voss et al. 2002].

O critério para seleção dos casos foi identificar organizações que buscavam a descentralização pela adoção do EUD há pelo menos 3 anos. Assim, seria possível analisar as estruturas existentes e experiências concretas de descentralização tanto do ponto de vista de organização quanto do ponto de vista dos usuários desenvolvedores. Foram projetados dois estudos caso holísticos, onde a unidade de análise é a organização.

Os principais métodos de coleta de dados foram entrevistas semi-estruturadas (Apêndice A), além de questionários e análise de documentos disponibilizados pelas organizações. Os dados foram coletados entre Setembro de 2016 e Setembro de 2017. As entrevistas buscaram investigar o cenário geral de descentralização por meio de EUD. As pesquisadoras têm experiência conduzindo entrevistas de campo no contexto científico, também com experiência em condução de entrevistas para elicitação de requisitos em empresas privadas. Na organização A, foram realizadas múltiplas entrevistas com dois profissionais líderes de área ao longo do período de coleta de dados. Na organização $\mathrm{B}$, foram realizadas múltiplas entrevistas com dois profissionais líderes de área, além de entrevista com 6 profissionais que atuam no desenvolvimento dos sistemas através de Oracle Apex (inicialmente destinado ao EUD).

Os questionários foram realizados após as entrevistas para detalhar ou confirmar dados obtidos nas entrevistas e foram enviados para a chefia da área responsável pela iniciativa EUD. Os documentos compreenderam, principalmente, site do processo, apresentações, relatórios e, portarias, em sua maioria documentos abertos ao público.

Os dados obtidos pelos três tipos de método de coleta foram triangulados durante a realização da análise. Quando havia dúvida sobre dados de entrevista ou documentos, ou mesmo conflito entre informações, novas entrevistas eram agendadas ou um questionário era enviado por e-mail.

O método de análise dos casos foi o de adequação ao padrão (pattern matching) [Trochim 1989], onde os padrões observados nos dados coletados (fundamentalmente empíricos) são comparados com os padrões previstos, ou seja, pelo framework conceitual proposto (Figura 1). Se os padrões coincidem, os resultados podem ajudar no aumento da validade interna do estudo. 


\section{Resultados}

Nesta seção os principais resultados dos estudos de caso são apresentados. A Tabela 1 descreve o perfil das organizações estudadas, onde cada organização é a unidade de análise do caso. Em seguida, os casos são descritos individualmente.

Tabela 1. Perfil das organizações

\begin{tabular}{|c|c|c|}
\hline Características & Organização (Caso) A & Organização (Caso) B \\
\hline Tipo & Pública & Pública \\
\hline Indústria & Governo & Governo \\
\hline $\begin{array}{l}\text { Estrutura } \\
\text { organizacional }\end{array}$ & Hierárquica & Hierárquica \\
\hline $\begin{array}{l}\text { Estrutura } \\
\text { organizacional de TI }\end{array}$ & $\begin{array}{l}\text { Departamento de TI e TI } \\
\text { departamental }\end{array}$ & $\begin{array}{l}\text { Departamento de TI e TI } \\
\text { departamental }\end{array}$ \\
\hline Tamanho da TI & $\begin{array}{l}136 \text { funcionários, } 79 \text { estagiários } \\
\text { e } 24 \text { terceiros }\end{array}$ & 153 funcionários e 4 estagiários \\
\hline $\begin{array}{l}\text { Descentralização por } \\
\text { EUD (Início) }\end{array}$ & 2007 & 2012 \\
\hline $\begin{array}{l}\text { Número atual de } \\
\text { pessoas envolvidas }\end{array}$ & $\begin{array}{l}\text { Departamento de TI: 3. Áreas } \\
\text { de Negócio: } 73 \text {. Estagiários: } 47\end{array}$ & $\begin{array}{l}\text { Departamento de TI: } 15 \text { pessoas } \\
\text { (10 alocados na TI corporativa e } \\
5 \text { nas áreas de negócio). Áreas } \\
\text { de Negócio: não há registro } \\
\text { (ocorrem por exceção). } \\
\text { Estagiários: inicialmente } 8 \text {, } \\
\text { atualmente } 0\end{array}$ \\
\hline $\begin{array}{l}\text { Perfil dos usuários } \\
\text { desenvolvedores }\end{array}$ & $\begin{array}{l}\text { Funcionários da área de negócio } \\
\text { com formação ou alguma } \\
\text { experiência prévia em TI. } \\
\text { Estagiários de TI. }\end{array}$ & $\begin{array}{l}\text { Funcionários da área de TI. } \\
\text { Estagiários de TI. }\end{array}$ \\
\hline $\begin{array}{l}\text { Perfil dos sistemas } \\
\text { desenvolvidos }\end{array}$ & $\begin{array}{l}\text { Sistemas cadastrais para } \\
\text { usuários internos e externos à } \\
\text { organização. }\end{array}$ & $\begin{array}{l}\text { Sistemas cadastrais para } \\
\text { usuários internos e externos à } \\
\text { organização. }\end{array}$ \\
\hline $\begin{array}{l}\text { Tecnologia de } \\
\text { desenvolvimento }\end{array}$ & Oracle Apex & Oracle Apex \\
\hline $\begin{array}{l}\text { Número de sistemas } \\
\text { desenvolvidos }\end{array}$ & mais de 200,141 em operação & 38 \\
\hline
\end{tabular}

\subsection{Caso A}

\subsubsection{Motivadores do usuário}

A organização A reconhece que sempre houve usuários desenvolvedores para soluções de problemas locais com ferramentas de escritório ou até mesmo com linguagens de programação. Um dos principais motivos é o desequilíbrio entre os recursos de TI disponíveis e a alta demanda das áreas clientes. 
Em 2007, com a priorização de um sistema corporativo, a organização reconheceu a iniciativa e participação das áreas de negócio na construção de soluções de TI departamentais e criou um programa baseado nos conceitos de EUD para apoiá-las. Foi definida uma abordagem EUD inicial para a organização, baseada na alocação de estagiários de cursos da área de computação pelas áreas de negócio que adotavam a abordagem EUD, para apoiarem a construção de sistemas de informação.

Com o movimento de descentralização da tecnologia pela adoção do EUD, qualquer área de negócio desenvolve soluções, seguindo os padrões estabelecidos pela Unidade EUD do departamento de TI. Caso contrário, será considerado TI Sombra, o que ainda ocorre na organização. Todas as ações e iniciativas de EUD no órgão são reguladas por uma política de Governança de TI.

Os funcionários com competências técnicas desenvolvem e podem contar com o apoio da Unidade EUD. Para apoiar os funcionários com pouco conhecimento técnico, estagiários de TI passaram a ser selecionados e treinados pela unidade responsável pelo EUD na organização. No entanto, a contratação continua sendo de responsabilidade das unidades de negócio. Essa estratégia é utilizada quando não há uma pessoa interessada ou apta em desenvolver aplicações.

Um dos entrevistados da organização comenta sobre o grau de empoderamento, flexibilidade e rapidez dos usuários evoluírem suas próprias aplicações:

"No caso de demandas de manutenção, a própria área desenvolvedora da solução fica responsável por implementar as alterações. Mudanças em sistemas devem ter a anuência do respectivo gestor da solução [...] temos visto que as aplicações desenvolvidas na ferramenta Oracle Apex são implementadas em intervalos de poucos minutos ou mesmo até três ou 4 anos, conforme a complexidade da aplicação [...] vejo que as unidades são satisfeitas com seus sistemas e ganham independência e facilidade para desenvolver e evoluir seus sistemas. Principalmente unidades que nunca eram priorizadas no Plano de TI"

Desde 2007, mais de 200 sistemas foram desenvolvidos nessa abordagem, sendo que cerca de 141 estão em operação.

\subsubsection{Questões Gerenciais e Contextuais}

Inicialmente, as soluções desenvolvidas por usuários desenvolvedores apresentavam qualidade questionável. Em geral, possuíam apenas o código-fonte, com indícios de falta de padrões, critérios de projeto ou testes de qualidade, o que comprometia a consistência dos resultados, assim como a segurança da informação.

O departamento de TI não participava ou não tinha conhecimento do desenvolvimento, que ocorria dada a dificuldade em atender as demandas pontuais das diversas áreas clientes. Quando as aplicações desenvolvidas por usuários finais precisavam ser evoluídas, em geral por necessidades do negócio, ou quando paravam de funcionar, muitas vezes a responsabilidade era transferida para o departamento de TI. A situação obviamente impactava o planejamento, assim como a produtividade, do departamento de TI, pois o escopo de atendimento crescia sem previsibilidade. 
Em 2007, a organização reconheceu que o desenvolvimento fora do controle do departamento de TI era um problema prioritário e decidiu adotar uma estratégia para lidar com o cenário. Foi incluída no Plano Diretor de Tecnologia uma iniciativa específica para lidar com desenvolvimento de sistemas departamentais. A iniciativa era centrada em prover ferramenta e suporte para usuários desenvolvedores.

Foi também criada, dentro do departamento de TI, uma unidade específica de governança e apoio ao desenvolvimento descentralizado. Ela visava garantir a qualidade, segurança e padronização das aplicações desenvolvidas pelos diversos departamentos, oferecendo um novo modelo de desenvolvimento.

Para lidar com a rotatividade de funcionários e estagiários, o departamento de TI intensificou a adoção de padrões e documentação. Em 2009, criou-se um núcleo de apoio ao desenvolvimento departamental cujo objetivo era reduzir o número de diferentes tecnologias no cliente, padronizar ainda mais as aplicações desenvolvidas por usuários e apoiar a continuidade e qualidade das soluções departamentais existentes. Por exemplo, o departamento de TI definiu o uso de um documento único, a Descrição Geral da Aplicação, focada na visão e nos requisitos do sistema. Com um documento simples, esperava-se incentivar uma atualização mais frequente. Em 2017, a organização ainda enxerga a rotatividade de desenvolvedores um desafio.

Estruturas de tomada de decisão. O departamento de TI define regras sobre como projetos devem ser conduzidos. Os sistemas são classificados quanto à abordagem de provimento (centralizado ou descentralizado) e quanto às características da solução (departamental ou corporativa). Projetos departamentais devem preferencialmente ser desenvolvidos no modelo descentralizado e projetos corporativos no modelo centralizado. No entanto, projetos corporativos podem ser desenvolvidos de forma descentralizada. É necessária uma aprovação do comitê gestor de TI.

As áreas de negócio, em parceria com o departamento de TI, avaliam a necessidade, pertinência e oportunidade do desenvolvimento da solução. Além disso, a padronização de processo de desenvolvimento descentralizado, infraestrutura e tecnologias para tal é exclusividade do departamento de TI. Cabe às unidades de negócio o poder de alterar os processos de trabalho que serão sistematizados pelo desenvolvimento.

Processos de alinhamento e Abordagens de comunicação Os riscos são gerenciados de maneira compartilhada. A área de negócio lida com riscos gerais na condução do projeto. Já o departamento de TI é responsável por evoluir o modelo de desenvolvimento descentralizado (através do serviço de apoio criado), e lidar com riscos associados à contratação de estagiários de TI e fornecimento de infraestrutura.

Um exemplo de risco identificado foi o de falhas na segurança de dados. O departamento de TI determinou que aplicações com funcionalidade muito críticas deveriam passar por um conjunto de testes antes de entrar em produção. Essa definição ocorreu em conjunto com as áreas de negócio.

Ao longo dos anos de experiência, o departamento de TI identificou a necessidade de criar algumas políticas para evitar riscos relacionados à segurança, desempenho, manutenibilidade, confiabilidade e acurácia das informações. Por exemplo, "usuários podem ter acesso às bases de dados corporativos, mas não podem gravar”. 
Outra política criada definiu que somente o departamento de TI poderia fornecer áreas de trabalho para utilização da ferramenta. Assim, a TI tem o controle das aplicações em desenvolvimento, com o objetivo de gerir e acompanhar com mais facilidade os usuários desenvolvedores. O departamento de TI monitora o ambiente acerca do consumo de recursos, como processamento e armazenamento, e para que boas práticas sejam seguidas.

A Tabela 2 resume as estruturas de tomada de decisão da organização A, parte das questões gerenciais.

Tabela 2. Estruturas de tomada de decisão do Caso A

\begin{tabular}{ll}
\hline Domínio & Tomada de Decisão \\
\hline $\begin{array}{l}\text { Alocação de } \\
\text { orçamento }\end{array}$ & Federada \\
\hline Gestão de demanda & Federada \\
\hline $\begin{array}{l}\text { Priorização de } \\
\text { projetos }\end{array}$ & Federada \\
\hline Alocação de pessoas & $\begin{array}{l}\text { Federado (TI recruta e treina } \\
\text { estagiários de TI/Área de } \\
\text { negócio contrata os estagiários e } \\
\text { aloca funcionários de negócio) }\end{array}$ \\
\hline Arquitetura & Centralizada \\
\hline Infraestrutura & Centralizada \\
\hline
\end{tabular}

\subsubsection{Fatores críticos de sucesso}

Tecnologia. A ferramenta principal de desenvolvimento escolhida para o desenvolvimento descentralizado foi a Oracle Application Express (ORACLE, 2017), mais conhecida como Apex. Ao conhecer a agilidade e simplicidade do desenvolvimento que a ferramenta possibilitava, a alta direção resolveu adotá-la, visto que o órgão já possuía uma licença Oracle e a ferramenta Oracle Apex poderia ser utilizada sem custos adicionais.

Um dos entrevistados avalia a facilidade de uso da ferramenta:

"[...] excelente ferramenta para desenvolvimento rápido de sistemas, com uma excelente curva de aprendizado e sem a exigência de profissionais com conhecimentos avançados em TI para o desenvolvimento de sistemas de menor complexidade e de uso cotidiano. Conforme o profissional vai se especializando ainda mais no uso dessa ferramenta e também nas tecnologias que a rodeiam, então sistemas mais robustos vão sendo desenvolvidos, fruto de uma evolução considerável das últimas versões de Apex que foram lançadas no mercado."

No entanto, também identifica limitações: 
"Nem toda unidade quer desenvolver seu sistema e a tecnologia Apex funciona apenas para sistemas web [...] difícil integração com dispositivos de hardware"

Em 2017, a organização ainda enxerga desafios relacionados à estabilização de sistemas desenvolvidos.

Treinamento. Para padronizar o desenvolvimento das aplicações, foram criados treinamentos para o Oracle Apex. O departamento de TI criou a infraestrutura necessária para hospedar o curso na Internet. Todos os usuários desenvolvedores passaram a realizar o treinamento antes de iniciar o desenvolvimento.

Suporte. Em 2009, foi criado um serviço de apoio ao EUD, que tinha o objetivo de gerenciar processos diversos do desenvolvimento descentralizado e apoiar os usuários desenvolvedores. Por exemplo, o grupo fornece o serviço de seleção de estagiários de TI para apoiar o desenvolvimento das aplicações EUD, sendo que a decisão é da área de negócio. O grupo também facilitava a identificação de novos projetos com complexidade alta para o departamento de TI. Aos poucos, o grupo foi conseguindo estabelecer sua função consultiva, não se envolvendo efetivamente no desenvolvimento e manutenção de sistemas.

A Figura 2 sintetiza os resultados do Caso A, em relação ao elementos apresentados no framework conceitual, assim como suas interações. Como elemento novo, ressalta-se a TI Sombra. Há também uma relação direta entre "Questões Gerenciais e Mecanismos de Governança"e "Treinamento, Suporte, Experts e Seleção de Estagiários", visto que o departamento de TI teve papel chave em priorizar recursos e estruturar tais ações. No framework conceitual inicial, esses dois elementos não estavam relacionados. O sombreamento em cinza ressalta o foco do departamento de TI, que tenta estabilizar priorizar os motivadores do usuário, enquanto garante parte do controle e governança.

\subsection{Caso B}

\subsubsection{Motivadores do usuário}

O desenvolvimento por usuário final iniciou em 2012, a partir da experiência do caso A. A principal motivação para a adoção do modelo naquele momento era prover uma ferramenta aos usuários, alternativa ao uso de planilhas, para resolver as demandas das áreas de negócio. Um dos entrevistados comenta:

"No início da adoção da metodologia os sistemas eram essencialmente departamentais, aqueles casos típicos de se sistematizar um pequeno controle geralmente feito em planilha."

Os funcionários da área de negócio usavam planilhas com grandes volumes de dados (planilhas de 250 colunas) para realizar suas atividades. A possibilidade de importar as planilhas em uma ferramenta de desenvolvimento rápido, sem entrar na fila do departamento de TI era muito animadora. Além disso, seria possível consultar o sistema central, onde os principais dados corporativos estão. Por fim, poderiam atualizar os dados de maneira concorrente, o que antes era impossível pelas planilhas. 


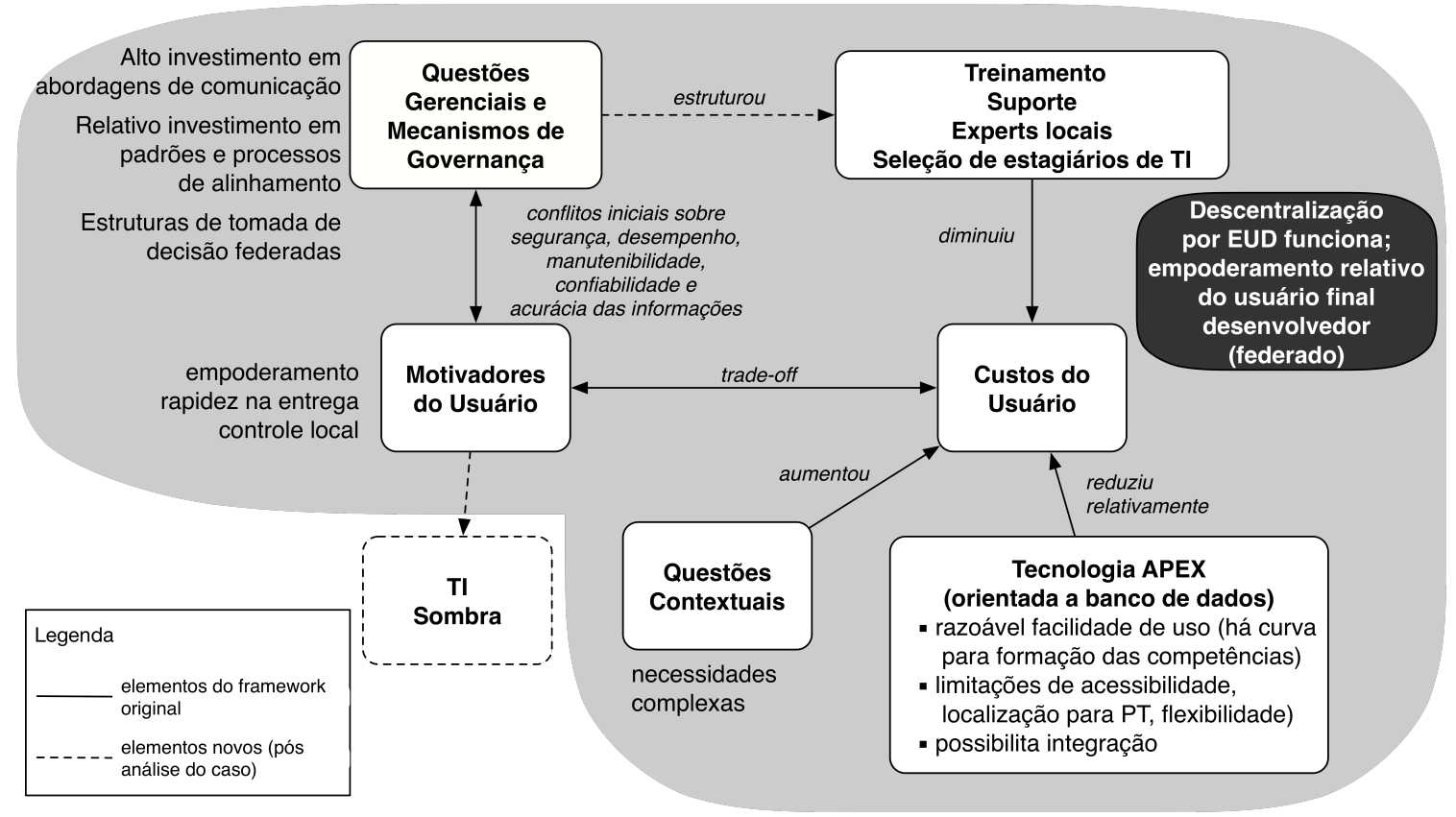

Figura 2. Síntese do Caso A

Inicialmente, havia três modalidades de desenvolvimento: desenvolvimento realizado por funcionário da organização $\mathrm{B}$, desenvolvimento realizado por estagiário alocado nas áreas de negócio e desenvolvimento por estagiário alocado na área de TI. O grupo de usuários-desenvolvedores era composto por dois funcionários da área de negócio e um estagiário de TI. Com o tempo, havia 8 estagiários que apoiavam diversos funcionários da área de negócio no desenvolvimento de aplicações. Um dos entrevistados afirma:

”cada analista responsável pela solução fica a cargo tanto da evolução como da sustentação. Como as necessidades ultimamente aumentaram muito, não é possível separar os papéis, e para cada sistema existe o funcionário responsável que acaba sendo o especialista naquele assunto. [...] quando há estagiário alocado na área, geralmente a sustentação e evolução são compartilhadas, ficando a gestão negocial e demandas mais complexas com o servidor, e o restante para o estagiário"

Atualmente, 19 áreas de negócio desenvolvem software nesse modelo. Há 10 funcionários do departamento de TI desenvolvendo soluções para 14 dessas áreas. Nas demais, há 5 funcionários responsáveis por sistemas, um em cada área. Essas 5 áreas demandam novos sistemas e correções muito rápidas, por isso o departamento de TI decidiu alocar seus funcionários diretamente na área negocial. Por falta de recursos, não há mais estagiários alocados nas áreas de negócio, apenas dentro da TI.

Um dos benefícios percebidos do modelo foi a velocidade de entrega de soluções. Era possível ter um sistema pronto em 6 meses, o que deixava os envolvidos satisfeitos pela sensação de atingimento de meta em um prazo razoável. O departamento de TI considera que a tecnologia APEX é uma solução que consegue atender a área fim do órgão, que vinha sendo pouco servida pela TI. Um dos entrevistados do departamento afirma: 
"Há muitos anos essas unidades vinham usando apenas o sistema processual da casa. A partir do momento que eles tiveram acesso a uma ferramenta que lhes deu certa independência, rapidamente aderiram. Antes disso, o departamento de TI nem conseguia atender esse tipo de demanda"

Desde 2012, 38 sistemas foram desenvolvidos nesse modelo e encontram-se em manutenção.

\subsubsection{Questões Gerenciais e Contextuais}

A partir da experiência do caso A, a organização B considerou que a gratuidade da licença da ferramenta Oracle/APEX, junto à baixa curva de aprendizagem, eram uma vantagem importante relacionada à custo-eficácia de iniciativa. No entanto, a organização não formalizou o desenvolvimento descentralizado. No Plano Diretor de TI há menções sobre iniciativas em Apex, principal ferramenta adotada na abordagem descentralizada, mas não descreve um processo de desenvolvimento para esse modelo.

Estruturas de tomada de decisão. O departamento de TI define regras sobre como projetos devem ser conduzidos. Todas as demandas de desenvolvimento de software do Órgão Público B são captadas e priorizadas de forma centralizada por equipes de análise técnica. A partir desse processo a demanda pode seguir por três caminhos: ser desenvolvida de forma centralizada pela TI tradicional, ser desenvolvida por funcionários de TI alocados na área de negócio na ferramenta APEX, ou ainda ser desenvolvida pelo departamento de TI (funcionário ou estagiário), utilizando APEX.

Os critérios para definir se uma demanda será desenvolvida no modelo EUD ou na TI centralizada levam em consideração fatores como a expectativa de tempo para atendimento da demanda, a complexidade da solução, a plataforma em que será utilizada a solução e a possibilidade da necessidade ser atendida por um software de mercado. É avaliado, de maneira informal, se a necessidade do usuário demandante será plenamente atendida com um sistema Apex, já considerando sua expectativa final da solução.

No início da metodologia de desenvolvimento descentralizado no Órgão Público $\mathrm{B}$, as demandas eram essencialmente departamentais de casos simples para sistematização um controle geralmente feito em planilhas. Com o tempo, as necessidades foram ficando mais complexas, necessitando de um acompanhamento mais próximo dos funcionários da TI. Atualmente, a tecnologia utilizada nesta metodologia, o Apex, tornou-se uma opção de tecnologia de sistemas assim como outras do desenvolvimento centralizado como Java e PHP. Um entrevistado afirma:

"Com o tempo, as necessidades foram ficando mais complexas, com muitas integrações, necessitando cada vez mais de um acompanhamento mais dedicado dos servidores que gerenciavam os estagiários. Atualmente, tornou-se uma opção de tecnologia de sistema da forma como são tratados Java e PHP aqui, ou seja, essa equipe de 10 servidores é responsável por sistemas que são sensíveis para o órgão [...] na grande maioria dos casos, sistemas nacionais" 
Um dos entrevistados comenta sobre a dificuldade de se estabelecer, de fato, o desenvolvimento por usuário final:

“Não dá pra ficar com o 'end-user' porque nem o que é mais centralizado, no estilo RAD, a gestão é madura"

É possível, no entanto, que alguns desenvolvimentos sejam iniciados na área de negócio, sem passar pelo processo de captação e análise centralizada, conhecido como TI Sombra. Nesses casos, o departamento de TI tomará conhecimento do sistema no momento em que for solicitada sua implantação em produção, uma vez que os usuários-desenvolvedores não possuem permissão para este procedimento.

O departamento de TI gerencia toda a infraestrutura necessária para o uso da ferramenta Apex, incluindo as necessidades relacionadas à administração dos dados. Sobre a gestão dos dados, um dos entrevistados comenta:

"Nossa equipe de administração de dados mantém o controle da criação de novas tabelas e demais objetos de banco, e recomenda que utilizemos dados e tabelas de esquemas corporativos sempre que possível [...] nem me lembro que tenha ocorrido de um sistema Apex solicitar alterações em modelos corporativos. Em geral, o esquema específico do sistema Apex fica com os dados típicos daquela necessidade, e os demais dados já existentes em outras bases são apenas consultados ou recuperados, sem redundância”

Não identificou-se um processo específico de monitoramento de uso da infraestrutura por esses sistemas. Um dos entrevistados narrou um caso de falha grave de uma aplicação desenvolvida nessa abordagem:

"Um select do mal derrubou o banco e todos os sistemas conectados ao banco de dados"

Processos de alinhamento. O departamento iniciou uma wiki com o processo para orientar os desenvolvedores que utilizam Apex. No entanto, esse site tornou-se desatualizado, conforme declarou um dos entrevistados:

"O que temos de formalização é a página da Wiki onde foi inicialmente detalhado o processo, mas que já não conta com atualização constante"

Abordagem de comunicação. O único canal oficial de comunicação identificado acerca da iniciativa de descentralização foo o Plano Diretor. No entanto, sem detalhamento ou outras comunicações de reforço, conforme menciona o entrevistado:

"No Plano Diretor há o detalhamento das iniciativas em Apex para o período, mas não há uma descrição do processo de desenvolvimento.

Também não há nenhuma publicação em política ou portaria."

A Tabela 3 resume as estruturas de tomada de decisão da organização B, parte das questões gerenciais. 
Tabela 3. Estruturas de tomada de decisão do Caso B

\begin{tabular}{ll}
\hline Domínio & Tomada de Decisão \\
\hline $\begin{array}{l}\text { Alocação de } \\
\text { orçamento }\end{array}$ & Centralizada \\
\hline Gestão de demanda & Centralizada \\
\hline $\begin{array}{l}\text { Priorização de } \\
\text { projetos }\end{array}$ & Federada \\
\hline Alocação de pessoas & $\begin{array}{l}\text { Centralizada (Funcionários e } \\
\text { Estagiários de TI) }\end{array}$ \\
\hline Arquitetura & Centralizada \\
\hline Infraestrutura & Centralizada \\
\hline
\end{tabular}

\subsubsection{Fatores críticos de sucesso}

Tecnologia. De acordo com os entrevistados, a ferramenta proporciona uma curva rápida de aprendizado e segurança. No entanto, um dos entrevistados comenta sobre dificuldades em relação à segurança da informação no uso da ferramenta selecionada (Apex):

"na versão 5 da ferramenta há bloqueio a alguns tipos de ataque que derrubavam o Apex na versão anterior como SQL injection”

Para muitos entrevistados, a ferramenta pode não parecer tão amigável para o usuário sem conhecimento de tecnologia. Além disso, à medida que as interfaces se tornam mais complexas, o desenvolvimento torna-se mais lento. Outra limitação mencionada por um dos entrevistados:

"os relatórios nem sempre ficam bom no Apex, nestes casos optamos por outras ferramentas especializadas”

Além do Apex, a organização B permite desenvolver em outras tecnologias. No entanto, é necessário que a área interessada obtenha uma autorização, a qual poderá ser emitida após a avaliação da tecnologia pela TI.

Treinamento. Para que estagiários da TI ou funcionários passem a utilizar o Apex, eles passam por um treinamento de duas semanas, realizado pela TI. Ao final desta capacitação, eles estão aptos a construir sistemas que fazer as operações básicas de inclusão, exclusão e alteração de registros em tabelas do banco de dados.

Cultura Durante as entrevistas sobre perspectivas futuras, os entrevistados consideram importante realizarem uma espécie de campanha de incentivo para sensibilizar e motivar os funcionários para EUD. Apontaram que não há ainda uma motivação intrínseca dos funcionários para se engajarem neste modelo, além da necessidade de reconhecimento pelo trabalho.

A Figura 3 sintetiza os resultados do Caso B, em relação ao elementos apresentados no framework conceitual, assim como suas interações. Como elemento novo, ressalta-se a TI Sombra, também resultado do Caso A. Há também uma relação direta entre "Questões Gerenciais e Mecanismos de Governança"e "Treinamento, 
Suporte, Experts e Seleção de Estagiários", visto que o departamento de TI não priorizou recursos para estruturar tais ações. Assim, não houve condições para sustentação da iniciativa de descentralização. A tecnologia adotada (Apex) passou a ser utilizada para desenvolvimento rápido de aplicações, ou Rapid Application Development (RAD). O sombreamento em cinza ressalta o foco do departamento de TI, que tenta estabilizar suas próprias questões gerenciais antes de priorizar os motivadores do usuário.

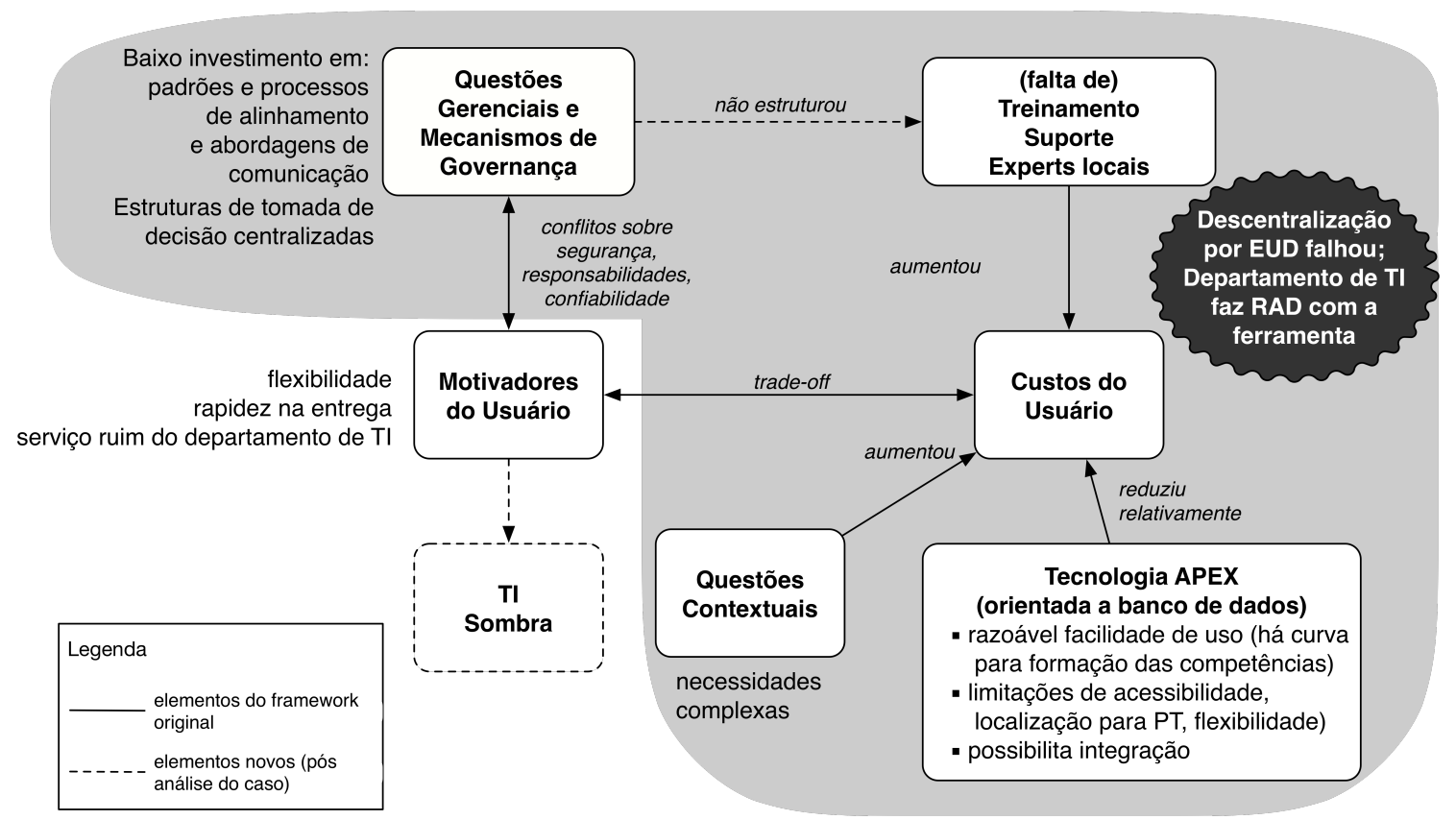

Figura 3. Síntese do Caso B

\section{Discussão e Limitações}

Em ambos os casos, como grande patrocinador do investimento, os departamentos de TI priorizam a otimização do modelo para favorecer seu próprio custo-eficácia, dado que antes não atendiam (ou atendia pobremente) muitas das áreas de negócio beneficiadas.

No caso A, a organização foi bem-sucedida em articular alguns dos fatores consideramos como críticos de sucesso para adoção de EUD [Fischer et al. 2004], o que permitiu avançar mais na descentralização da função de TI do que no caso B. A organização A priorizou e fez investimentos ao longo de 10 anos para assegurar treinamento e suporte ao desenvolvimento por usuário final, além de estabelecer claramente pelo menos dois mecanismos de governança: estruturas de tomada de decisão e abordagens de comunicação.

No caso B, os funcionários não consideram a gestão da TI centralizada madura suficiente, tornando-se um passo difícil estruturar um maior nível de descentralização. O modelo, inicialmente pensado para o usuário desenvolvedor, acabou sendo adaptado para uso do próprio departamento de TI, que passou a desenvolver soluções rápidas com a ferramenta selecionada (Apex) para áreas de negócio. Com o passar do tempo, o modelo tornou-se uma alternativa para o desenvolvimento centralizado, sem necessidade de times grandes ou terceirização. Assim, o departamento de TI do caso B atende mais 
departamentos do que antes, porém sem empoderar o usuário das áreas de negócio, o que limita o alcance do que pode ser feito através da tecnologia.

O resultado sugere que a organização primeiro precisa aprender a gerir prioridades e demandas de TI de maneira centralizada, para que seja possível discernir o quê e como descentralizar funções de TI para outros departamentos e pessoas. Também sugere que a abordagem EUD pode acabar tornando-se um RAD quando as questões gerenciais e mecanismos de governança não estão suficientemente maduros para dar suporte à adoção da tecnologia pelo usuário final. A ferramenta de apoio ao desenvolvimento por usuário final passa a ser usada pelo próprio departamento de tecnologia para o desenvolvimento rápido de aplicações (RAD). Assim, uma iniciativa EUD pode acabar sendo distorcida ou transformar-se em mais uma forma de desenvolvimento centralizado, agora apoiado por ferramentas originalmente voltadas para usuários finais.

Não houve evidências no caso B de que há motivadores suficientes para gerar condição de aprendizado para usuários aprenderem novas tecnologias providas pela organização. Os dados apontam que a organização falhou em criar condições para essa motivação em maior escala. Em ambos os casos, houve participação ativa de funcionários e estagiários com conhecimento prévio em TI. Não houve evidência de que pessoas sem esse conhecimento anterior se interessassem por aprender a tecnologia para desenvolver sistemas.

Os riscos de segurança, a complexidade de algumas demandas em termos de número de integrações, assim como a otimização do modelo para custo-eficiência para o departamento de TI são forças centralizadoras. Em ambos os casos, a segurança foi um dos fatores importantes para maior centralização do modelo, confirmando estudos anteriores sobre EUD e TI Sombra [Panko and Port 2013]. Mesmo as melhorias relacionadas a ataques por injeção pela nova versão da ferramenta Apex, usada em ambas organizações, não modificaram a visão das organizações sobre esse grau de centralização.

De fato, estudos apontam que, em geral, organizações falham em endereçar a visão do usuário sobre segurança, assim como seu comportamento e conhecimento básico em como prevenir problemas com dados sensíveis [Gross and Rosson 2007]. Há limitações de usabilidade nas próprias ferramentas oferecidas aos usuários, que induzem a erros e falhas subsequentes. Além disso, o usuário final pode lidar com questões básicas de segurança quando há treinamento, conscientização, monitoramento e motivação [Stanton et al. 2005].

Ambas organizações adotaram a mesma tecnologia, considerada adequada para EUD em termos de curva de aprendizado e possibilidades de integração. No entanto, a ferramenta ainda assim requer treinamento e, em ambas organizações, o usuário desenvolvedor, em geral, tem conhecimento prévio de TI. A ferramenta Apex foi avaliada quanto a 11 características favoráveis ao EUD [Melo et al. 2017]. Os resultados demonstram que a ferramenta tem limitações de usabilidade, em particular, acessibilidade, localização para português, flexibilidade e competências necessárias para manipulação. Além disso, a ferramenta é voltada para um domínio específico de aplicações que consultam e atualizam dados armazenados em bancos de dados relacionais [Richardson and Rymer 2016].

Os resultados indicam que em ambos os casos ainda ocorre TI sombra. Esse 
resultado confirma estudos que afirmam que sistemas sombra persistem ou retornam, ainda que haja esforços para sua eliminação [Walters 2013, Fürstenau et al. 2017]. No caso A, mesmo com investimento de 10 anos no aprimoramento da descentralização, ainda há espaço para investigação dos motivadores de usuário que levam à adoção de outros processos e tecnologias para construção de soluções.

A situação pode ter sido originada de um conflito entre a gestão e as motivações dos usuários, que gostariam de maior empoderamento, como também por um trade-off relacionado ao custo de aprendizado, programação e depuração da ferramenta pelo usuário. No entanto, apenas futuros estudos poderão confirmar essas hipóteses.

Importante ressaltar que a TI sombra também é vista como fonte de inovação em muitas organizações [Györy et al. 2012], não sendo necessariamente um problema a ser eliminado, mas um fenômeno a ser compreendido, principalmente pelo potencial de aumento de produtividade, flexibilidade e inovação dirigida por usuário [Silic and Back 2014, Zimmermann et al. 2017].

Nesse contexto, é importante notar que não houve menção nas entrevistas, no questionário ou nos documentos analisados sobre qualquer perspectiva de inovação por trás das iniciativas de descentralização por EUD, como sugere a literatura da área [Niehaves et al. 2012, Köffer et al. 2014, Andriole 2015, Bygstad 2016]. Embora algumas organizações já invistam na criação de infraestruturas que empoderem o usuário desenvolvedor dentro e fora da organização [Angeli et al. 2014, Bygstad 2016], os casos estudados não demonstram essa visão da função de TI. Há infraestrutura disponível, mas não prevê suporte a uma gama ampla e heterogênea de aplicações desenvolvidas pelo usuário desenvolvedor, de modo a garantir flexibilidade e empoderamento.

Além disso, em ambos os casos não foi identificado um monitoramento do valor gerado pela abordagem além da maior vazão de sistemas sob mecanismos de redução de riscos. Assim, pode não haver ainda um motivo claro para avançar na descentralização da TI por EUD, potencialmente atingindo modelos de governança descentralizados ou participatórios.

Este estudo possui ameaças à validade inerentes a estudos de caso: validade de construto, interna, externa e confiabilidade [Yin 2009]. Em relação à validade de construto, triangulou-se três tipos de fontes de evidência, além de discussões entre o time de pesquisa. Em relação à validade interna, procurou-se estabelecer relações causais através de correspondência de padrões entre o framework conceitual proposto e os padrões observados nos dados. As relações não observadas não foram descritas nos resultados (Figuras 2 e 3), assim como elementos e relações novos foram adicionados de forma destacada. Para aumentar a validade externa dos resultados, estudamos duas organizações com razoável similaridade de intenções para que fosse possível contrastar resultados. Por fim, o instrumento de coleta de dados é apresentado de forma a aumentar a confibilidade do procedimento adotado.

A principal limitação do estudo foi baixa quantidade de dados provenientes de usuários-finais desenvolvedores não participantes das iniciativas oficiais de descentralização. Essa perspectiva certamente enriqueceria a compreensão do sistema socio-técnico estudado, assim como circunstâncias que levam à existência de TI sombra. 


\section{Conclusão}

A maior parte do software já é escrito por usuários finais que precisam cumprir objetivos de negócio através da TI, embora a governança estabelecida pelas organizações nem sempre seja adequada. A compreensão de como organizar a descentralização da função de TI para usuários finais, de modo a preparar a organização para os desafios e oportunidades do século 21, torna-se chave.

Nesta pesquisa apresentou-se dois estudos de caso em organizações que buscavam descentralizar a função de TI através de EUD. Dentre os resultados analisados por meio do framework conceitual proposto, destaca-se que a maturidade prévia em governança da TI foi fator importante na capacidade de descentralização através de EUD, embora ambos casos não tenham atingido alto grau de descentralização. Além disso, a TI sombra permaneceu em ambos os casos. Ambas organizações não demonstraram buscar inovação com as iniciativas de descentralização, embora essa seja uma visão fundamental da função de TI no século 21 .

No contexto de governança de TI em plena economia digital, expandir a investigação sobre a dinâmica das tensões de poder entre o departamento de TI e os usuários finais desenvolvedores é uma questão relevante, dado que a TI vem tornando-se cada vez mais pervasiva e de fácil acesso aos usuários [Fürstenau et al. 2017]. Como trabalho futuro, existem diversas avenidas possíveis de pesquisa. Estudar mais organizações buscando compreender a descentralização e empoderamento de usuários. Outro caminho interessante é aprofundar a compreensão das jornadas de aprendizado e trabalho do usuário-final desenvolvedor para compreender os caminhos entre EUD e TI Sombra. Compreender a interação de usuários e tecnologias de suporte a EUD é de extrema relevância, já que elas podem viabilizar a entrada de mais usuários desenvolvedores. Por fim, uma linha interessante de investigação é mapear e investigar que infraestruturas de governança participatória vêm sendo propostas, uma vez que elas podem viabilizar o controle (automatizado) sem sacrificar o empoderamento de usuários e até mesmo possibilitar o envolvimento ativo de stakeholders externos à organização.

\section{Referências}

Andriole, S. J. (2015). Who owns IT? Communications of the ACM, 58(3):50-57.

Angeli, A. D., Bordin, S., and Blanco, M. M. (2014). Infrastructuring participatory development in information technology. In Participatory Design Conference, PDC'14, Windhoek, Namibia, October 6-10, 2014, Volume 1 - Research Papers, pages 11-20.

Barker, S. and Fiedler, B. (2011). Developers, decision makers, strategists or just end-users? redefining end-user computing for the 21st century: A case study. Journal of Organizational and End User Computing, 23(2):1-14.

Benbasat, I., Goldstein, D. K., and Mead, M. (1987). The case research strategy in studies of information systems. MIS Quarterly, 11(3):369-386.

Bharadwaj, A., Sawy, O. A. E., Pavlou, P. A., and Venkatraman, N. (2013). Visions and voices on emerging challenges in digital business strategy. MIS Quarterly, 37(2):633-661. 
Bradley, R. V., Byrd, T. A., Pridmore, J. L., Thrasher, E., Pratt, R. M., and Mbarika, V. W. (2012). An empirical examination of antecedents and consequences of IT governance in us hospitals. Journal of Information Technology, 27(2):156-177.

Bygstad, B. (2016). Generative innovation: a comparison of lightweight and heavyweight IT. Journal of Information Technology, 32(2):180-193.

Chua, C., Storey, V., and Chen, L. (2014). Central IT or shadow IT? factors shaping users' decision to go rogue with IT. In Myers, M. D. and Straub, D. W., editors, ICIS. Association for Information Systems.

DeLone, W., Migliorati, D., and Vaia, G. (2017). Digital IT Governance, pages 205-230. Springer International Publishing, Cham.

Dittrich, Y., Bolmsten, J., and Eriksson, J. (2017). End User Development and Infrastructuring - Sustaining Organizational Innovation Capabilities, pages 165-206. Springer International Publishing, Cham.

Ferneley, E. (2007). Covert end user development: A study of success. Journal of Organizational and End User Computing, 19(1):62-71.

Figueiredo, R. M. d. C., Venson, E., Melo, C. d. O., Ramalho, C., Modesto, A. S., Melo, L. S. d., Nobre, A. J., Moraes, J. M., Ferreira, M. H. P., and Martins, P. (2017). Desenvolvimento pelo usuário final: conceitos, estratégias e casos de adoção: relatório de pesquisa. Technical report, Universidade de Brasília.

Fischer, G. (2009). End-user development and meta-design: Foundations for cultures of participation. In End-User Development, 2nd International Symposium, IS-EUD 2009, Siegen, Germany, March 2-4, 2009. Proceedings, pages 3-14.

Fischer, G., Giaccardi, E., Ye, Y., Sutcliffe, A. G., and Mehandjiev, N. (2004). Meta-Design: A Manifesto for End-User Development. Communications of the ACM, 47(9):33.

Frey, T. and Buxmann, P. (2011). The importance of governance structures in IT project portfolio management. In 19th European Conference on Information Systems, ECIS 2011, Helsinki, Finland, June 9-11, 2011, page 17.

Fürstenau, D., Rothe, H., and Sandner, M. (2017). Shadow systems, risk, and shifting power relations in organizations. Communications of the Association for Information Systems (CAIS), 41:3.

Gross, J. B. and Rosson, M. B. (2007). Looking for trouble: Understanding end-user security management. In Proceedings of the 2007 Symposium on Computer Human Interaction for the Management of Information Technology, CHIMIT '07, New York, NY, USA. ACM.

Györy, A., Cleven, A., Uebernickel, F., and Brenner, W. (2012). Exploring the shadows: IT governance approaches to user-driven innovation. In 20th European Conference on Information Systems, ECIS 2012, Barcelona,Spain, June 10-13, 2012, page 222.

Haes, S. D. and Grembergen, W. V. (2009). An exploratory study into IT governance implementations and its impact on business/IT alignment. Information Systems Management, 26(2):123-137. 
Karimi, J., Bhattacherjee, A., Gupta, Y. P., and Somers, T. M. (2000). The effects of mis steering committees on information technology management sophistication. Journal of Management Information Systems, 17(2):207-230.

Ko, A. J., Abraham, R., Beckwith, L., Blackwell, A., Burnett, M., Erwig, M., Scaffidi, C., Lawrance, J., Lieberman, H., Myers, B., Rosson, M. B., Rothermel, G., Shaw, M., and Wiedenbeck, S. (2011). The state of the art in end-user software engineering. ACM Computing Surveys (CSUR), 43(3):21:1-21:44.

Köffer, S., Fielt, E., and Niehaves, B. (2015). IT consumerization and its effects on IT business value, IT capabilities, and the IT function. In 19th Pacific Asia Conference on Information Systems, PACIS 2015, Singapore, July 5-9, 2015, page 169.

Köffer, S., Ortbach, K., and Niehaves, B. (2014). Exploring the relationship between IT consumerization and job performance: A theoretical framework for future research. CAIS, 35:14.

Lieberman, H., Paternò, F., Klann, M., and Volker, W. (2006). End-User Development: An Emerging Paradigm. Springer Netherlands, 1st edition.

Maceli, M. G. (2017). Tools of the Trade: A Survey of Technologies in End-User Development Literature, pages 49-65. Springer International Publishing, Cham.

Melo, C., Moraes, J., Ferreira, M., and da Costa Figueiredo, R. M. (2017). A method for evaluating end-user development technologies. In 23rd Americas Conference on Information Systems, AMCIS 2017, Boston, MA, USA, August 10-12, 2017.

Niehaves, B., Köffer, S., and Ortbach, K. (2012). IT consumerization - A theory and practice review. In 18th Americas Conference on Information Systems, AMCIS 2012, Seattle, Washington August 9-11, 2012.

Panko, R. R. and Port, D. N. (2013). End user computing: The dark matter and dark energy of corporate IT. J. Organ. End User Comput., 25(3):1-19.

Peterson, R. (2004). Crafting information technology governance. EDPACS, 32(6):1-24.

Ping-Ju Wu, S., Straub, D. W., and Liang, T.-P. (2015). How information technology governance mechanisms and strategic alignment influence organizational performance: insights from a matched survey of business and it managers. MIS Quarterly, 39(2):497 $-\mathrm{A} 7$.

Richardson, C. and Rymer, J. R. (2016). The Forrester Wave: Low-Code Development Platforms.

Silic, M. and Back, A. (2014). Shadow IT - a view from behind the curtain. Computers \& Security, 45:274-283.

Stanton, J. M., Stam, K. R., Mastrangelo, P., and Jolton, J. (2005). Analysis of end user security behaviors. Computers \& Security, 24(2):124-133.

Trochim, W. M. (1989). Outcome pattern matching and program theory. Evaluation and Program Planning, 12(4):355 - 366. Special Issue: The Theory-Driven Perspective.

von Hippel, E. (2005). Democratizing Innovation. MIT Press. 
Voss, C., Tsikriktsis, N., and Frohlich, M. (2002). Case research in operations management. International Journal of Operations \& Production Management, 22(2):195-219.

Walters, R. (2013). Bringing IT out of the shadows. Network Security, 2013(4):5-11.

Weill, P. and Ross, J. (2004). IT Governance: How Top Performers Manage IT Decision Rights for Superior Results. Harvard Business School Press, Boston, MA, USA.

Weill, P. and Ross, J. (2005). A matrixed approach to designing IT governance. 46:26-34.

Winkler, T. J. and Brown, C. V. (2013). Horizontal allocation of decision rights for on-premise applications and software-as-a-service. Journal of Management Information Systems, 30(3):13-48.

Yin, R. K. (2009). Case Study Research: Design and Methods. Applied Social Research Methods. SAGE Publications.

Zimmermann, S., Rentrop, C., and Felden, C. (2014). Managing shadow IT instances - A method to control autonomous IT solutions in the business departments. In 20th Americas Conference on Information Systems, AMCIS 2014, Savannah, Georgia, USA, August 7-9, 2014.

Zimmermann, S., Rentrop, C., and Felden, C. (2016). Governing identified shadow IT by allocating IT task responsibilities. In 22nd Americas Conference on Information Systems, AMCIS 2016, San Diego, CA, USA, August 11-14, 2016.

Zimmermann, S., Rentrop, C., and Felden, C. (2017). A multiple case study on the nature and management of shadow information technology. Journal of Information Systems, 31(1):79-101. 


\section{Apêndice A - Protocolo de Entrevista}

Explicações gerais sobre o objetivo da entrevista:

< explicação dos objetivos - atentar para não enviesar

Explicar como estamos definindo EUD e Desenvolvimento descentralizado>

Permissão para gravação:

Antes de começar a entrevista, você tem alguma pergunta?

\begin{tabular}{l|l|}
\hline Roteiro de Entrevista & $\begin{array}{l}\text { Notas de pesquisa (observações } \\
\text { durante a entrevista) }\end{array}$ \\
\hline $\begin{array}{l}\text { Entrevistador(a): } \\
\text { Data da entrevista: }\end{array}$ & \\
\hline
\end{tabular}

Dados demográficos

Entrevistado(a):

Organização:

Número de funcionários:

Indústria: Governo/

Área/Departamento:

Número de funcionários da área/depto:

Papel/Cargo:

Formação:

Experiência profissional:

\section{Visão geral de EUD na Organização/Departamento}

1. Qual foi a motivação para a adoção de EUD?

2. Quais áreas usam ferramentas EUD? Que necessidades/problemas elas têm para o uso de EUD (ex: automatizar operação, analisar dados, gerar relatório)? Qual o critério para escolher o desenvolvimento descentralizado?

3. Que ferramentas são usadas? Qual o modelo de contratação (ex: assinatura, licença, software livre)?

4. Houve algum tipo de capacitação/treinamento para usar essas ferramentas? E outros tipos de investimento?

5. Como é o apoio da organização/departamento para esse tipo de atividade? (aguardar resposta e explorar cenários onde há reconhecimento ou problemas)

6. O que melhorou na organização com a adoção dessa abordagem? Quais as evidências?

7. Os objetivos iniciais foram alcançados? Você poderia contar um cenário onde isso ocorreu? (explorar caso de sucesso, motivador de venda para outros adotarem)

8. Você poderia contar um cenário onde EUD não deu certo? (explorar caso de dor e muito aprendizado)

\section{$<$ Fechamento da entrevista $>$}

